\title{
Benchmark
}

\section{Use of Adenoviral Vectors with a Minimal Cytomegalovirus Promoter}

BioTechniques 34:600-602 (March 2003)

\section{Andreu Ferrer-Martínez, Celia García-Martínez, and Anna M. Gómez-Foix Universitat de Barcelona, Barcelona, Catalonia, Spain}

E1-deleted recombinant adenoviruses 5 are widely used as gene vectors for basic and clinical research. Most adenoviral constructions bear an expression cassette inserted into the E1 region of their genome close to the $5^{\prime}$ end and include a heterologous promoter. The major immediate early human cytomegalovirus (hCMV) and the Rous sarcoma virus promoters, which have fairly high and consistent activity, are among the most extensively used. Nevertheless, expression of delivered protein from these promoters is often excessive, and weaker transcriptional activity would be desirable. Adenoviral sequences located $5^{\prime}$ of the site of insertion, in the region between nucleotides 22 and 341, contain a number of identified enhancer elements (the left ITR and the E1A enhancer) that, in certain conditions, may have an intrinsic promoter activity or may modulate the activity of inserted heterologous promoters (1-4). At $3^{\prime}$ of the insertion site there are enhancer sequences such as that of the right ITR and those present in the E2 and E4 promoters, which, although quite distal, can also interfere. This concept is supported by the inclusion of insulator sequences from the chicken $\gamma$-globin locus flanking the expression cassette (4), which abolished the background expression of a metalinducible promoter and increased the response to inducible factors.

Therefore, characterization of the influence of adenoviral sequences on weak heterologous promoters is of high interest for the design of new adenoviral vectors. The objective of this study was to reveal the activity of these viral sequences, in the context of hepatic and muscle cells, using the minimal region of the major immediate early hCMV promoter. Two adenoviruses were prepared: AdMIN and AdCMV. AdMIN included a minimal hCMV promoter also used by Baron et al. (5) (-31 to +16 nucleotides from the start transcription site) cloned at nucleotide 455 of adenoviral genome in the backbone of plasmid pACCMVpLpA (6), followed by the reporter gene EGFP. In AdCMV, which was prepared for comparison purposes, the immediate early hCMV promoter $(-806$ to +16 nucleotides from the start transcription site) was cloned instead of MIN. Adenoviruses were generated by standard techniques (6) and used to transduce several immortalized hepatic cells including FTO2B, H4IIE, Hep3B, HepG2, and primary cultured rat hepatocytes. Gene delivery to cell cultures was accomplished by exposing cells to viruses for $2 \mathrm{~h}$ at a MOI of 80 to ensure high efficiency of transduction. Infections were always performed in parallel with both viruses on each cell type. After two days of culture, cells were scraped in Tris buffer, sonicated, and centrifuged at $7400 \times g$ for $15 \mathrm{~min}$. Protein aliquots (1.5 and $15 \mu \mathrm{g}$ ) of the supernatants were run on a SDS-polyacrylamide gel and transferred into hydrophobic filters. Ponceu S staining was routinely used to confirm proper protein loading and uniform sample transfer to the membrane. EGFP immunodetection was performed by regular Western blot procedures. In AdCMV-transduced cells transcriptional activity of the hCMV promoter proved to be high, although slight differences were observed among cell types. In the H4EII cell line and primary cultured hepatocytes, protein levels were about half of those in the rest of the cells (Figure 1). When AdMIN was used to express EGFP, much higher variability was detected. EGFP levels were negligible in rodent hepatoma cells, FTO2B and H4IIE, but significant in human hepatic cell lines and rat hepatocytes. In these cells, however, transcriptional activity from AdMIN was about 10 times lower than that from AdCMV (Figure 1).

Expression of EGFP from AdMIN and AdCMV was also tested in primary cultured human muscle cells (obtained with informed consent, Hospital Vall d'Hebron, Barcelona) and in the C2C12 immortalized rodent cell line. Myoblasts and myotubes were analyzed four days after adenoviral transduction. In all these cells, both myoblasts and differentiating myotubes, EGFP expression was high when using the AdCMV construction (Figure 2). In contrast, EGFP protein levels were barely detectable in $\mathrm{C} 2 \mathrm{C} 12$ cells and absent in primary cultured muscle cells transduced with AdMIN.

The differences in AdCMV activity 
are attributable to transcription factors, which are present in variable amounts and states depending on the cell type and differentiation stage. Several cellular transcription factors, including NF$\kappa 0 \mathrm{~B} / \mathrm{rel}$, CREB/ATF, AP1, retinoic acid receptor, SP-1, serum response factor, and ELK-1, have been reported to stimulate the major immediate early hCMV enhancer $(7,8)$. Nevertheless, the response of the AdMIN should be explained in terms of the activity of the MIN promoter as an enhancer trap of adenoviral elements, likely those proximal sequences located at the $5^{\prime}$ end of the virus, since AdMIN lacks the hCMV enhancer. These elements are $(i)$ the ITR, which contains conserved sequence motifs that bind the cellular transcription factors, such as NFIII/OCT-1,
SP1, and ATF (9), and (ii) element I of the E1A enhancer, which binds the cellular protein EF-1A (10).

In conclusion, the MIN promoter in the context of the E1 region of adenovirus is active in hepatic cells, whereas it shows low to null activity in muscle cells. Its differential response provides useful information regarding the cell-dependent interactions of adenoviral elements with weak heterologous promoters inserted in the E1 region and brings up the need to take this into account when designing new adenoviral vectors. On the other hand, we show that the AdMIN system has significant advantages to drive the expression of a transgene. First, the small size of the MIN promoter provides additional space for the insertion of larger

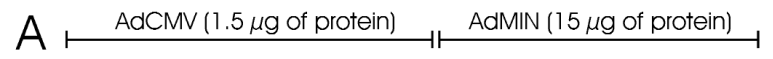
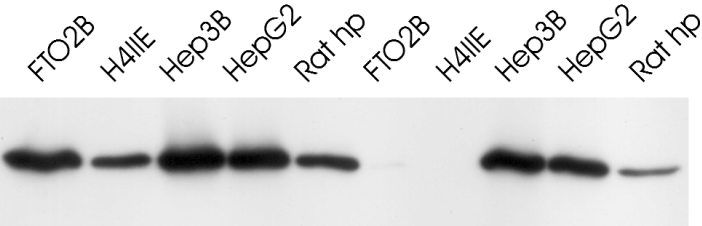

B

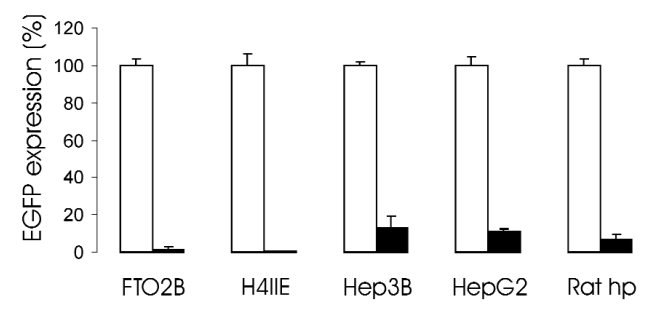

C

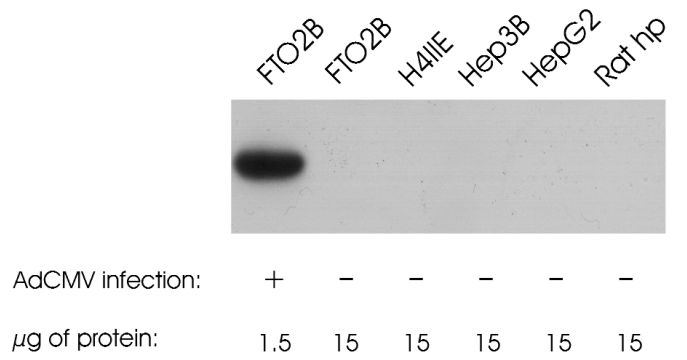

Figure 1. EGFP expression in adenovirus-transduced cells of hepatic origin. Four immortalized cell lines (FTO2B, H4IIE, Hep3B, and HepG2) and primary cultured rat hepatocytes (Rat hp) were infected with AdCMV or AdMIN and cultured for two days. Experiments were repeated 3-5 times. Protein (1.5 and $15 \mu \mathrm{g}$ ) from the AdCMV- and AdMIN-transduced cells, respectively, was used in Western blot analyses to detect EGFP expression. (A) Representative Western blot. (B) Densitometric analysis of data expressed as a percentage relative to each AdCMV-transduced cell type ( $\overline{\mathrm{x}} \pm \mathrm{SEM})$. (C) EGFP detection in non-transduced and AdCMV-transduced cells. 


\section{Benchmark}

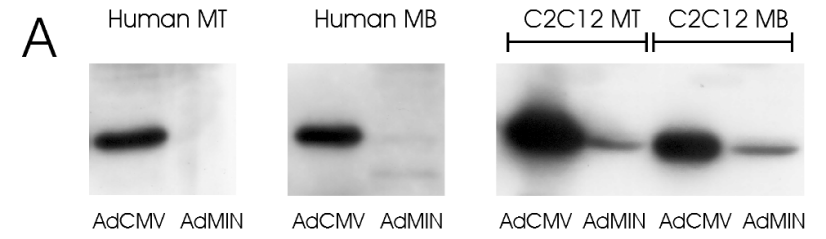

$\mathrm{B}$
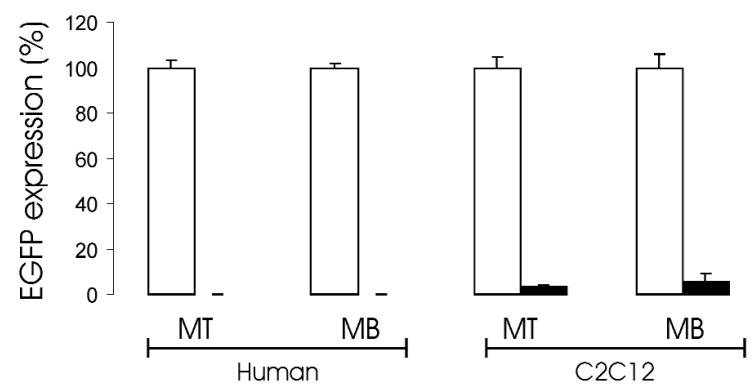

C

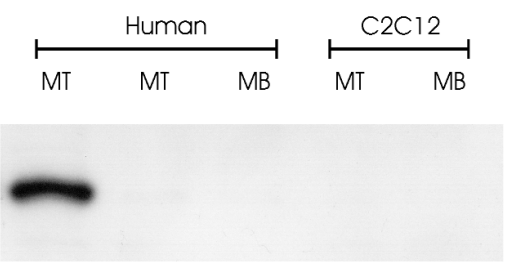

AdCMV infection:

$\mu \mathrm{g}$ of protein
$+$

$1.5 \quad 15$
15

$15 \quad 15$

15

Figure 2. EGFP expression in adenovirus-transduced cells of muscle origin. Primary cultured human muscle cells and $\mathrm{C} 2 \mathrm{C} 12$ cells were infected with AdCMV or AdMIN and cultured for four days. Experiments were repeated three times. Protein $(1.5$ and $15 \mu \mathrm{g})$ protein from the AdCMV- and AdMIN-transduced cells, respectively, was used in Western blot analyses to detect EGFP expression. (A) Representative Western blot. (B) Densitometric analysis of data expressed as a percentage relative to each AdCMV-transduced cell type ( $\overline{\mathrm{x}} \pm$ SEM). (C) EGFP detection in non-transduced and AdCMV-transduced cells. MT, myotube; MB, myoblast.

transgene constructs. Second, the AdMIN vector has weak transcriptional activity, unlike AdCMV, whose activity is so high that the levels of protein generated can be excessive for certain purposes. This AdMIN characteristic allows one to increase the multiplicity of viral infection, reaching higher efficiency in terms of the number of transduced cells without excessive transgene expression.

\section{ACKNOWLEDGMENTS}

This work was supported in part by grant no. SAF2000-0193 from the Di- rección General de Investigacion, Ministerio de Ciencia y Tecnología (Spain). A.F.-M. is the recipient of a fellowship from the Dirección General de Investigación, Ministerio de Ciencia y Tecnología (Spain).

\section{REFERENCES}

1.Babiss, L., J.M. Friedman, and J.E. Darnell, Jr. 1986. Cellular promoters incorporated into the adenovirus genome: effects of viral regulatory elements on transcription rates and cell specificity of albumin and $\beta$-globin promoters. Mol. Cell. Biol. 6:3798-3806.

2.Hatfield, L. and P. Hearing. 1993. The NFIII/OCT-1 binding site stimulates adenovirus DNA replication in vivo and is functionally redundant with adjacent sequences. J. Virol. 67:3931-3939.

3.Shi, Q., Y. Wang, and R. Worton. 1997. Modulation of the specificity and the activity of a cellular promoter in an adenoviral vector. Hum. Gen. Ther. 8:403-410.

4.Steinwaerder, D. and A. Lieber. 2000. Insulation from viral transcription regulatory elements improves inducible transgene expression from adenovirus vectors in vitro and in vivo. Gene Ther. 7:556-567.

5.Baron, U., S. Freundlieb, M. Gossen, and H. Bujard. 1995. Co-regulation of two gene activities by tetracycline via a bidirectional promoter. Nucleic Acids Res. 23:3605-3606.

6.Becker, T.C., R.J. Noel, W.S. Coats, A.M Gómez-Foix, T. Alam, R.D. Gerard, and C.B. Newgard. 1994. Use of recombinant adenovirus for metabolic engineering of mammalian cells. Methods Cell Biol. 43:161189.

7.Chan, Y., C.J. Chiou, Q. Huang, and G.S. Hayward. 1996. Synergistic interactions between overlapping binding sites for the serum response factor and ELK-1 proteins mediate both basal enhancement and phorbol ester responsiveness of primate cytomegalovirus major immediate-early promoters in monocyte and T-lymphocyte cell types. J. Virol 70:8590-8605.

8.Meier, J. and J.A. Pruessner. 1996. Regulation of human cytomegalovirus immediateearly gene expression. Intervirology 39:331342.

9.Hatfield, L. and P. Hearing. 1991. Redundant elements in the adenovirus type 5 inverted terminal repeat promote bidirectional transcription in vitro and are important for virus growth in vivo. Virology 184:265-276.

10.Bruder, J.T. and P. Hearing. 1989. Nuclear factor EF-1A binds to the adenovirus E1A core enhancer element and to other transcriptional control regions. Mol. Cell. Biol. 9:5143-5153.

Received 17 October 2002; accepted 25 November 2002.

Address correspondence to:

Dr. Andreu Ferrer-Martínez

Departament de Bioquímica i Biologia

Molecular

Universitat de Barcelona

Martí i Franquès 1

08028, Barcelona, Catalonia, Spain

e-mail:aferrer@porthos.bio.ub.es 